\title{
Empleo de redes sociales y aplicaciones digitales en la estrategia comunicativa multipantalla de los principales grupos audiovisuales privados en España
}

\author{
María Julia GonZÁLEZ CONDE \\ Universidad Complutense de Madrid \\ mariajul@ucm.es \\ Carmen SAlgado SANTAMaríA \\ Universidad Complutense de Madrid \\ carmen.salgado@ucm.es
}

Recibido: 16 de julio de 2015

Aceptado: 18 de septiembre de 2015

\begin{abstract}
Resumen
La importancia del uso de las redes sociales en los medios de comunicación ha provocado que los grandes grupos audiovisuales privados españoles, Atresmedia y Mediaset, centren su interés en este nuevo cauce de interactividad, que les beneficia como plataforma digital de distribución creando sus propias cuentas en las redes sociales más visitadas e incluyendo la opción de que los usuarios compartan sus contenidos a través de ellas. Una potenciación de servicios interactivos online que hace multiplicar su presencia en estos otros espacios de participación, incrementando su tráfico de visitas y mejorando su alcance con el efecto cadena entre los miembros de la red social y sus medios de comunicación. Este artículo muestra los enfoques, planteamientos particulares y medidas estratégicas comunicativas recogidos de las entrevistas personales realizadas a los principales gestores de las redes o community managers de cada uno de estos dos grupos audiovisuales analizados, así como expone los contenidos programáticos que ofrecen sus distintos canales digitales; dando especial énfasis a aquellos sistemas interactivos que focalizan su atención a través de las multipantallas, principalmente del dispositivo móvil.
\end{abstract}

Palabras clave: Redes sociales, Plataforma digital, Dispositivos Móviles, Mediaset, Atresmedia.

\section{Use of social networking and digital applications in the communication strategy of the largest private multi-media groups in Spain}

\begin{abstract}
The impact of the use of social networks in the most important media has caused many changes, like private groups, Atresmedia and Mediaset, focus their interest in this new channel of interactivity, as digital distribution platform. This companies create own accounts on the most popular social networks that includes the option to users to share their content through them. The growth of online interactive services multiplies its presence in these spaces of participation and increases their traffic and improves the scope of the chain between the members of the social network and its mass media. This article presents the particular approaches and communication strategic measures as a result of personal interviews have been made to the community managers of each of these two audiovisual groups. Also it's important to know the programmatic content that offer in different digital channels; with special emphasis on those interactive systems that focus your attention through the multi-screen, especially mainly from the mobile device.

Keywords: Social Network, Online Platform, Mobile Device, Mediaset, Atresmedia.

\section{Referencia normalizada}

GONZÁLEZ CONDE, María Julia y SALGADO SANTAMARÍA, Carmen (2015): "Empleo de redes sociales y aplicaciones digitales en la estrategia comunicativa multipantalla de los principales grupos audiovisuales privados en España”. Estudios sobre el Mensaje Periodístico. Núm. especial diciembre, págs.: 97-108. Madrid, Servicio de Publicaciones de la Universidad Complutense.
\end{abstract}


Sumario: 1.Introducción. 2. Metodología. 3. Desarrollo; 3.1. Atresmedia Digital. Se generan nuevos contenidos para sus nuevos soportes y aplicaciones; 3.2. Atresmedia y su convergencia en las redes sociales; 3.3. Mediaset. Uso y perfil de sus cinco aplicaciones y plataformas digitales; 3.4. Mediaset. Nuevas experiencias con su público interactivo y sus redes sociales. 4. Conclusiones. 5. Referencias bibliográficas.

\section{Introducción}

La innovación tecnológica y el desarrollo de la digitalización han abierto una ventana de oxígeno para los grupos audiovisuales españoles, propuestos en nuestro estudio, debido a la variedad de canales temáticos que han podido agrupar en cada uno de ellos. Canales generalistas, especializados o temáticos que conforman el espectro televisivo privado $^{1}$ en el ámbito de las plataformas nacionales de la televisión convencional. "[...] Una lucha encarnizada de las cadenas por la conquista de este televidente fraccionado y compulsivo, que ansía distintos tipos de combinado de realidad, ficción y entretenimiento" (González y Barceló, 2009: 25)

El principal valor de uso de las redes sociales se fundamenta en que sirven como herramientas de distribución ${ }^{2}$ donde se conjugan todos los elementos que componen la naturaleza de internet, lo que acentúa su singularidad en el sistema comunicativo. Un significado que no se explicaría si no se tuviera en cuenta el proceso de un nuevo entorno tecnológico, dentro de la era de la participación, del Periodismo 3.0., en el que se está experimentando, entre otros cambios, una nueva realidad comunicativa e informativa que subyace junto a la práctica tradicional de sus canales y medios tradicionales. "La televisión actual como hecho social, a la vez que un lenguaje con estilo propio, sigue considerándose el gran discurso o libro abierto de la cultura de nuestro tiempo" (González, 2009c: 175)

Expondremos algunas de las características que avalan el uso de las redes sociales como su aplicación multimedia simplificada, su servicio de hipertexto, con enlaces para conectar informaciones o páginas web entre sí; su posibilidad de lograr una comunicación múltiple con un mayor estadio de interactividad o la determinación de renovación o actualización de contenidos por parte del usuario, en el momento que él elija, entre otras muchas utilidades.

Los medios reconocen estas ventajas de servicio y requieren a las redes sociales como una excelente técnica de difusión y distribución de sus propios contenidos. Un añadido que corresponde a un progreso natural, fomentado en los procesos de convergencia y distribución multipantallas, en los que la decisión de demanda del espectador o usuario, se antepone a cualquier otro pronóstico o predisposición programática. Se habla de una audiencia prominentemente activa que se caracteriza por la "fragmentación y la especialización" (Domingo, Salaverría, Cabrera y Aguado, 2007). Una población que, a diferencia de la más joven, debe pasar por distintas etapas de adaptación en un entorno donde las TIC son omnipresentes y se han convertido en ele-

1 Y que en estudios posteriores ampliaremos al sector público.

2 Consideramos que, a pesar de que las redes sociales no son medios de comunicación, sí son nuevos canales de distribución que permiten enlazar sus contenidos en función de lo que publique su audiencia. De esta manera, cada usuario configurará un perfil propio o participará en una puesta en común interactiva para crear la portada de la red. 
mentos naturales, integrados en la idiosincrasia de la realidad cotidiana, donde se acumulan y saturan los flujos informativos de viejas y nuevas tecnologías. (González, 2009a: 131) Asimismo, la digitalización ha impulsado la multiplicación de canales temáticos, presentes en sus distintas plataformas de emisión ininterrumpida, capaz de aglutinar multitud de contenidos, sobre todo de carácter lúdico y de entretenimiento.

Hablamos de una revolución comunicativa, cultural, además de tecnológica encabezada por una población adolescente -joven, entre los 10 a los 25-30 años-, especialmente impaciente y dispersa, que se encuentra a la vanguardia de una actividad frenética, fruto de un contacto constante y con la posibilidad de acceso a contenidos infinitos, que además está vinculada al uso de multipantallas y por tanto al valor de la interactividad como uno de sus principales retos. (Barceló et al, 2014: 33).

Asimismo, en el tema de las redes sociales, constatamos que los casos en los que predomina la construcción de identidad, éstas son consideradas como servicios de la web donde se permite compartir información, coordinar acciones y en general, mantenerse en contacto todos sus usuarios. Coincidimos con Orihuela y Salaverría en señalar que las redes sociales son una prolongación de las relaciones personales en la vida real, y "[...] son asimismo, paradigma de las posibilidades que nos ofrece esta nueva forma de usar y entender internet" (Salgado y González, 2013: 60)

\section{Metodología}

Los resultados del presente estudio corresponden a la tercera fase (2014-15) de una investigación más amplia, perteneciente al proyecto nacional de I+D+i titulado "Cybermedia II: Desarrollos e Innovaciones del Periodismo en las Redes sociales en Internet y Telefonía móvil. Convergencias, Modelos de negocios, Servicio y Formación" (Plan Nacional I+D+i Ref. CSO2011-25235), cuyos resultados ya han sido publicados en el libro Innovaciones periodísticas en las redes sociales (2012) y Contenidos y servicios periodísticos en las redes sociales (2013).

En este capítulo concreto, se plantea una investigación de carácter descriptivo, que parte de un modelo empírico y de contextualización general de análisis sobre el desarrollo y la implicación de la cultura de red en las actuales labores de planificación y estrategia mostrada por los principales grupos audiovisuales privados, Mediaset España y Atresmedia. En el contexto y desarrollo de la investigación empírica se realizaron entrevistas personales a los máximos responsables de los grupos audiovisuales referidos, como periodistas gestores de las redes ${ }^{3}$ (o community manager), para conocer de primera mano los requisitos y aspectos particulares tenidos en cuenta por las empresas de comunicación en la presentación de contenidos y diseño de canales digitales y servicios interactivos de valor añadido, lo que nos ayudará a entender mejor su proyecto comunicativo y el resultado del mismo.

3 José Manuel González Pacheco (Director general en Atresmedia Digital) y Francisco Sierra Hernando (Director de contenidos en Antena 3 Multimedia) del Grupo Atresmedia. Sonia Got Leibar (Coordinadora de Redes Sociales en Mediaset) y María Sainz (Coordinadora Apps de Mediaset) del Grupo Mediaset España. 
El carácter descriptivo de la investigación hizo que las entrevistas se efectuaran en un momento concreto a los dos grupos con el fin de determinar su opinión y posicionamiento en el mismo periodo de tiempo. La información recogida de los representantes de Atresmedia (semana del 19 al 23 de enero de 2015) y Mediaset (semana del 26 al 30 de enero de 2015). Todas las entrevistas fueron grabadas y tuvieron un carácter distendido, dialoguista y abierto, sin estar sujetas a un horario determinado, aunque en su comprobación constatamos que existía una media de dos horas cada una.

Sin embargo, el cuestionario sí estuvo fijado a un modelo formal, planteándose las hipótesis en formato de preguntas (30) y buscando sus respuestas; y dividido en tres grandes bloques, que se encierran en estos enunciados generales:

- Aspectos técnicos/plataformas utilizadas/modelos comunicativos

- Contenidos (formatos y géneros) y relación con la audiencia (los jóvenes)

- Aplicaciones digitales, sistemas expresivos, servicios de valor añadido, interactivos y multimedia

Tras esta auscultación cualitativa se procedió a la recopilación sistemática de las informaciones recogidas vinculadas a nuestro principal objetivo de análisis y las conclusiones derivan de su verificación tras la observación de los distintos canales señalados.

De este modo, nuestro objeto de estudio se basa en la observación, planteamiento, análisis y descripción de las dinámicas de relación que se generan en las redes sociales y que son utilizadas en los medios digitales privados, cadenas de TV y canales en la web, de los grupos Atresmedia y Mediaset España como estrategia de búsqueda de mercados de contenidos especializados para lograr nichos específicos de audiencia. No se analizarán las redes como producto interactivo entre los usuarios de los medios tanto como su valor de multiplicidad de opciones y contribución en la organización programática de estos grupos mediáticos, que se ven obligados a una nueva orientación, dispuesta no sólo a un esfuerzo de difusión y dinamización, a través de las redes sociales de mayor penetración Twitter y Facebook, sino a la mejora e incremento de la conexión entre los usuarios, así como a ampliar y compartir los procesos y modelos comunicativos. Cada uno de estos grupos, de manera similar y singular, intenta potenciar sus características y funciones, cuando se vincula a una u otra red social tanto desde la perspectiva de expresión como de capacidad de acrecentar y optimizar sus posibilidades de navegación, hipertextualidad-hipermedialidad e interactividad con su audiencia. (Cebrián Herreros ${ }^{4}$ )

La exposición de resultados según los elementos de análisis que se han mencionado obedece a una razón práctica ya que supone una aportación, desde el punto de vista periodístico y de comunicación, sobre el establecimiento, evolución y apuntes de tendencia en el funcionamiento, estructura, contenidos y públicos de las principales ca-

4 Recogido del libro Contenidos y servicios periodísticos en las redes sociales (2014), in memoriam del profesor Cebrián, coordinado por Jesús Flores, cuyo primer capítulo "El periodismo entre la convergencia e interconexiones de las redes sociales" había sido escrito por aquél insigne investigador. Los datos se recogen en el apartado de Referencias bibliográficas. 
denas televisivas nacionales privadas que ahora se encuentran en las plataformas activas de cada uno de los principales grupos audiovisuales privados, que operan en nuestro país.

Una vez justificada nuestra investigación y habiendo expuesto aquellos aspectos y datos relevantes relacionados con el objeto de estudio, su finalidad y metodología, desarrollamos a continuación los aspectos más destacados que constituyen parte de una muestra orientativa sobre la estrategia comunicativa llevada a cabo por los community manager en cuanto a su valoración, opinión y conformación de sus canales televisivos en relación con su público a través de las redes sociales, aplicaciones, plataformas y servicios interactivos integrados.

\section{Desarrollo}

\subsection{Atresmedia Digital. Nuevos contenidos para sus nuevos soportes y aplicacio- nes}

Atresmedia uno de los principales grupos de comunicación españoles que está presente en los principales medios de comunicación: televisión, radio, cine, internet y publicidad, desarrolla entre sus líneas de negocio, Atresmedia Digital, que aglutina la oferta digital del Grupo, a la vanguardia de las nuevas tecnologías, y es en este ámbito en el que atrae el interés en nuestra investigación.

Atresmedia apostó por el carácter interactivo de la televisión hace cinco años, cuando inició el proyecto "Ant3.0.", con el que se pretendía, en primer lugar, dar respuesta a la demanda de sus espectadores, conscientes del cambio en la forma de ver la televisión; y después, generar espacios propios dentro del entorno digital, que no se encuentran en la programación televisiva convencional.

De entre todas las aplicaciones que ha desarrollado en cada uno de sus portales, tanto para programas como para dispositivos en movilidad, destacan tres aplicaciones estrellas: "Atresplayer", "Atresmedia Conecta" y la más reciente, "Neox Kidz".

Fue en Octubre de 2013, cuando se lanza "Atresplayer", una plataforma donde se concentran las señales en directo de toda su programación y el catch up, es decir, todos los archivos que generan los canales del Grupo. En la actualidad esta aplicación ya ha alcanzado un volumen de descargas que supera los cuatro millones tanto para tabletas como para smartphones. Y todo bajo un mismo concepto, que los usuarios puedan encontrar los contenidos que ofrece el Grupo a través de las plataformas o dispositivos que quieran, televisión interactiva, tabletas o teléfonos móviles.

Con el lanzamiento del "Modo Salón" en su plataforma "Atresplayer", se puede visionar el contenido completo de las grandes series, que se supone que son las que más impactan a los telespectadores 6 . Asimismo, a través del "Modo Salón Premium", se ofrecen servicios de pago a través de internet, para acceder a series y estrenos de alta

5 Lo que los responsables de Atresmedia denominan, la "joya de la corona".

6 El criterio tradicional de audiencia se ve afectado por el planteamiento del número de seguidores y número a los que se sigue dentro del canal, lo que exige otras estrategias de actuación de los medios en las redes en ese conjunto activo de hábitos, tiempos, contenidos y contactos. 
calidad y sin publicidad. También se tiene acceso ilimitado a todo catálogo de Antena 3 , con más de dos mil capítulos de series, Tv movies y programas.

Por otra parte, "Atresmedia Conecta" es la aplicación interactiva de segunda pantalla que permite disfrutar de contenido extra e interactuar durante la emisión en directo de las principales series y programas de la cadena, favoreciendo la eliminación de barreras entre el medio televisivo y su audiencia. Gracias a su second screen, que conecta en tiempo real los contenidos de la televisión en directo, con teléfonos inteligentes y tabletas, se acercan los temas allí donde se encuentren los espectadores. Así, al mismo tiempo que se está visionando un programa en la pantalla del televisor, se puede estar recibiendo contenido extra en forma de preguntas, imágenes o videos. El nivel de interactividad de esta aplicación es elevado al alcanzar los quinientos mil usuarios y no sólo para votaciones o encuestas, sino para contenidos adicionales que no se visualizan en la tele, como alguna toma falsa, adelanto de capítulos, fotografías, estadísticas o puntuaciones en el transcurso, por ejemplo, de una competición de Fórmula 1.

Asimismo, una de las aplicaciones gratuitas más recientes (diciembre de 2014) es "Neox Kidz". Está orientada a un público infantil y basada en los conceptos de entretenimiento, educación, seguridad y usabilidad. Su descarga en tabletas y móviles da acceso a la nueva web infantil del Grupo Atresmedia, que ofrece todo el contenido de "Neox Kidz" y una selección de los mejores programas del resto de cadenas del grupo. Las ventajas que aporta esta aplicación es que ofrece contenidos exclusivos, estrenos, videos y juegos online, más allá de lo que se emite en televisión con el objetivo de que los niños jueguen y aprendan.

Con un carácter completamente independiente del que se genera en las cadenas, Atresmedia continúa haciendo frente al reto de la creación de contenidos propios en el ámbito digital, con dos líneas muy claras de actuación en torno a una temática vertical o transversal y a una política multichannel dentro de YouTube. En los últimos meses, se han generado diversidad de contenidos verticales, como "Celebritis", "Se estrena", "Cocinatis", "Tecnología", "CienciaXplora", "TecnoXplora", "Correr y Fitness", o "Bienestar", entre otros. Son contenidos temáticos independientes, sólo para internet, que pueden cruzarse con los grandes portales del grupo: A3, La Sexta, Neox y Nova.

En cuanto a las producciones audiovisuales propias y dentro de la política de $\mathrm{mul}$ tichannel, basada en YouTube, se crean espacios cortos fundamentalmente de humor, orientados a un usuario joven y a un consumo internacional.

A pesar de que todavía la utilización de contenido audiovisual en todos los soportes complementarios es minoritario, crece respecto al consumo tradicional de televisión. Por este motivo, el papel estratégico de "Atresmedia Digital", según su director general, José Manuel González Pacheco, consiste en estar bien posicionado dentro de la nueva fórmula de distribución de su programación y que sirva, al mismo tiempo, de complemento de los contenidos tradicionales. "Para cada producto se generan entornos digitales a través de making off, spin off, encuentros digitales, contenido transmedia, video encuentros, etc. y el contenido de cada cadena marca el contenido de los entornos digitales". De este modo, a través de todos sus medios, de la publicidad, 
y de modelos de pago emergentes que se están explotando, se conseguiría hacer marketing, promocionar programas y fidelizar a sus audiencias.

Además, como nos indica el director de contenidos de Atresmedia, Francisco Sierra, se observa una nueva tendencia. El consumo de contenidos televisivos en movilidad, bien en tabletas o en smartphones, crece exponencialmente al ritmo en el que se presentan en el mercado productos que permiten nuevas funcionalidades. Por lo que respecta a Atresmedia, se puede decir que algunos de sus programas ya tienen un consumo cercano al 50/50 y las últimas cifras señalan que ese crecimiento es imparable. Si seguimos las estadísticas que reconocen que dos terceras partes de los espectadores que ven la televisión están utilizando un dispositivo móvil, éste es un dato determinante a tener en cuenta por parte de estos responsables para rediseñar o crear nuevos contenidos complementarios al programa televisivo. Lo que también obligará a adaptarse a los nuevos usos y hacer que en estos soportes exista una mayor navegabilidad. Siempre teniendo en cuenta que no todas las pantallas pueden englobar el mismo contenido, ya que los propios terminales pueden limitar su uso y consumo.

Así, existen determinados formatos como son los videos cortos o videoclips que funcionan mejor en los smartphones, mientras que las tabletas son idóneas, por ejemplo para consumos de programas completos, series, concursos o espacios informativos.

Todas estas innovaciones tecnológicas han provocado también la definición de nuevos tipos de usuarios en función de las diferentes formas de acceder a los contenidos. En concreto, en Atresmedia, existen tres tipos de usuarios: el Anónimo, el Registrado y el Premium, llamado éste último al espectador de pago. Usuarios que de la misma manera varían en función del lugar en el que se encuentren, dentro o fuera del territorio español; lo que requiere un acoplamiento a las distintas fórmulas -la gratuita, de registro o de pago- e igualmente supone la necesidad de familiarizarse con los diversos estados que incluyan una determinada programación.

\subsection{Atresmedia y su convergencia en las redes sociales}

Las redes sociales, fundamentalmente en Facebook y Twitter, juegan un papel protagonista en relación a la convergencia de este mundo social con las aplicaciones, contenidos y programas que ofrecen las cadenas de televisión. En este sentido, Atresmedia se define como el grupo de comunicación de España con mayor número de seguidores en Facebook y Twitter, por encima de la cifra de los veintiocho millones. Entre estos seguidores destacan los de "El Hormiguero" el programa español en emisión con más seguidores en las redes sociales.

En ese sentido, Twitter es la red social más empleada para los comentarios en tiempo real sobre temas de actualidad y noticias, mientras que en Facebook la actividad se desarrolla en series y programas a lo largo de la semana. Programas como "El Intermedio" tienen un impacto muy fuerte en redes sociales al contar con audiencias muy fieles y participativas.

La presencia de las redes sociales y su protagonismo han llegado hasta el punto de ser considerados un elemento más en la producción de los guiones de programas televisivos en los que se incluyen los hashtag o las propias redes. 
Además, existen datos cuantitativos que reflejan que el éxito de programas televisivos como "Tu cara me suena" también tiene su réplica en las redes sociales, donde semanalmente se reúnen sus seguidores, llegando a acumular en sus once primeras galas alrededor de ciento noventa trending topics nacionales y ciento catorce mundiales.

"Como nuestro producto estrella son las series es normal que tengamos un mayor consumo de catch up, más que en otros productos digitales interactivos en los que se haya generado escenarios $360^{\circ}$ o transmedia, pero también en internet funcionan muy bien los entornos digitales, por ejemplo, de los Reality"7.

\subsection{Mediaset. Uso y perfil de sus cinco aplicaciones y plataformas digitales}

El grupo Mediaset España produce y emite contenidos audiovisuales en distintas plataformas (televisión e internet). Su actividad se centra esencialmente en la producción y exhibición de contenidos televisivos en sus seis principales canales: Telecinco, Cuatro, Factoría de Ficción, Boing, Divinity y Energy. Asimismo, cuenta en la web con Telecinco.es, Cuatro.com, Divinity.es y Mitele.es.

Sin embargo, nuestro interés se va centrar en las cinco aplicaciones que son considerados por Mediaset de gran transcendencia para el Grupo. Nos referimos a "Mitele", "La Voz", "Gran Hermano", "MotoGP" y "Mediasetsport".

De todas ellas, la aplicación estrella es "Mitele" al contar con unos componentes que la confieren mayor transversalidad. Su estructura y finalidad se asemeja a una videoteca en donde se pueden ver los directos, "el don de mandos" o los diferidos, contenido a la carta y un poco de cine de pago. Esta aplicación además de ser gratuita y de incorporar cine "Premium", apuesta por la interactividad. Durante los programas se lanzan las preguntas interactivas, con un llamamiento desde la tele, de acuerdo a un contenido amplio para los usuarios y como acompañamiento del visionado o complemento del contenido de los programas.

En la aplicación de "Gran Hermano" la interactividad se ajusta a este programa determinado ${ }^{8}$. Con ella, el usuario contesta a preguntas, resuelve situaciones que están ocurriendo en el programa y al mismo tiempo desde el canal se decide lo que se va a contar sobre los avatares de Facebook y se informa de la gente que en ese instante se encuentra interactuando. Su fórmula representa mayor interactividad y realismo del momento inmediato y del acercamiento con la audiencia. Además, la expresión de voto representa un gran atractivo para su público que, al mismo tiempo, se puede contemplar en la pantalla ("la tele te ve a ti"). Se trata de una proyecto piloto que ya había sido probado meses antes con otro programa, en "Supervivientes" y viendo el éxito alcanzado se adaptó y se hizo más extenso en la aplicación de "Gran Hermano". En un futuro cercano esta experiencia se quiere ajustar a una mayor transversalidad e incorporarla a "Mitele", su app estrella con más de tres millones de descargas.

7 Entrevista al Sr González Pacheco de Atresmedia (20 de enero de 2015)

8 Esta aplicación interactiva, que lleva ejecutándose desde 2014, está muy considerada por el Grupo por su inmejorable funcionamiento y gran interés de la audiencia, según nos expresa su responsable María Sainz. 
Mediaset apostó mucho por su primera aplicación relacionada con el deporte, "Mediasetsport", que surgió precisamente a raíz del Mundial de Brasil de fútbol. Se creó con el ánimo de poder mantenerse en activo incluso después de que finalizara éste, pero no alcanzó ningún impacto debido al nefasto resultado del equipo español, lo que hizo que tuviera que ser reconvertida para el Mundial de Basket y en estos momentos, se utiliza para todos los partidos en directo, como, por ejemplo los de fútbol sala, entre otros, salvo a lo concerniente a carreras de motos que se desarrollan en su propia aplicación, en la "MotoGP". Una aplicación específica que cuenta con un nicho muy importante debido a que los moteros son usuarios muy fieles a este deporte. No es la aplicación que más descargas tiene, en ningún caso comparable con "Mitele", pero según sus responsables la fidelidad de su público hace que merezca la pena apostar por el deporte y las motos.

En cuanto a las aplicaciones para programas en directo, los que mejor funcionan son los Reality, como por ejemplo el "24 horas del Gran Hermano", lo que no ocurre con programas de actualidad o con los informativos. Por ello, Mediaset está ideando vías de interactuación (intervenciones directas en debates) para este tipo de público.

La audiencia de Mediaset es muy variada, aunque tiende a ser mayoritariamente masculina, femenina o joven de acuerdo al programa o aplicación concreta que se trate. En el caso de "Mitele", su público es muy diverso.

Los programas de mayor interactividad son los Realities, sobre todo "Gran Hermano" y "Supervivientes", aunque han mostrado interés por otros géneros, como el de ficción. Fue una primera experiencia que se llevó a cabo en el canal Cuatro, en una serie titulada "Ciega a citas", donde el espectador elegía a su personaje favorito e interactuaba personalmente con él, como en un WhatsApp; así podían mantenerse conversaciones previas sobre diferentes temas, como el vestuario que iba a lucir el actor o actriz u otras cuestiones relacionadas con su actuación, decisiones que podía o no tomar, o sobre su comportamiento en la serie.

Existen contenidos extras en los programas en donde se realiza interactividad. Por ejemplo en "Gran Hermano", en "Gran Hermano Vip" y en "Supervivientes", cuando la gran gala empieza antes en la aplicación. Para ello, hay un grupo de periodistas que se denominan "periodistas interactivos" que van por los pasillos del recinto televisivo (de Telecinco) captando instantáneas y exclusivas.

En cuanto al hábito de visionado de las aplicaciones, el televisor sigue siendo, para la audiencia general de Mediaset, el soporte elegido, aunque a la hora del "don de mando", del diferido, la gente prefiere verlas a través del móvil. De cualquier manera, el público parece optar antes por aquella aplicación, que ha sido diseñada exclusivamente para el dispositivo móvil, que por la que ha sido adaptada a otra pantalla.

\subsection{Mediaset. Nuevas experiencias con su público interactivo y sus redes sociales}

"No nos importa arriesgar", aparenta ser el lema de todo este Grupo. En estos momentos uno de los últimos proyectos que se está llevando a cabo está relacionado con la conexión directa de los dispositivos móviles al receptor de televisión ("Conectar con la tele"), ya que se han registrado picos de tráfico alrededor de medianoche, sobre las 11 de la noche, como un momento elegido por la gente para "trastear" con su aparato 
móvil, desde lugares, como el dormitorio, donde quizá no se encuentre ningún receptor televisivo.

En cuanto al uso de las redes sociales, éstas parecen ser la mejor forma de conocer a los espectadores, sobre todo teniendo en cuenta el valor añadido que representan para el medio. ${ }^{9}$ Social media origina que la televisión sea mucho más entretenida, se enriquezca más y produzca mayores impactos sociales.

Además, para los responsables de este Grupo las redes sirven fundamentalmente en programas en directo, de actualidad o entretenimiento, para testar la opinión, para escuchar a la audiencia, para saber lo que realmente funciona y lo que no. Este es el caso, por ejemplo de "Todo va bien", un programa de entretenimiento, en directo, que depende mucho de lo que ocurra en las redes. Por nuestra parte observamos que este aspecto no siempre es coincidente, porque, aunque existan casos aislados, en la mayoría de las ocasiones el paso lo sigue marcando la televisión ya que muchos de los asuntos cobran fuerza cuando se ven en la pequeña pantalla.

Mediaset tiene cerca de veintiséis millones de seguidores, en las redes sociales, y un total de quinientas cuentas. Sin embargo, comprobamos que en ellas también figuran programas que en ese mismo momento no se encuentran en antena. Este hecho se justifica, según sus responsables, cuando existe la posibilidad de volver a emitirse en un futuro cercano; o bien, casos particulares como el programa anual de "la Voz" que, mantiene sus redes sociales activas durante más tiempo por la gran variedad de contenidos que éstas presentan (espacios musicales, información reciente o comentarios sobre los participantes o novedades sobre sus coaches).

De todas esas cuentas adscritas a las redes, se hallan algunas muy potentes en $\mathrm{Fa}-$ cebook, que incluso superan el millón de seguidores ${ }^{10}$. Es el caso de "la Voz", de "Gran Hermano" o de "Mujeres y hombres y viceversa".

En cuanto a la selección de Twitter o Facebook las dos herramientas merecen la atención de Mediaset, según el objetivo pretendido o del tiempo que se disponga para el mismo. Este grupo reconoce que Twitter tiene la gran ventaja de ser una red más "abierta" con posibilidad de retorno y de una lectura rápida, por lo que y es más utilizada para conocer lo que la gente opina, mientras que el consumo de Facebook al ser más reposado, funciona mejor, en las llamadas a la acción, para la participación en concursos, el acceso a un video o a la Web.

\section{Conclusiones}

Verificamos que aunque la mayoría de los medios pertenecientes a los dos Grupos audiovisuales analizados están presentes en casi todas las redes sociales, como Instagram, LinkedIn, Google+ o Pinterest, sus máximos esfuerzos se concentran en Facebook y Twitter, las redes más extendidas en España. De este modo, advertimos que la prioridad se encuentra en la selección y presencia en las redes fijas y en las

9 "Leemos más que escribimos", nos confía Sonia Got, coordinadora general de Redes sociales, de Mediaset.

10 "La que se avecina" fue la primera serie que superó el millón de seguidores en Facebook, según datos ofrecidos por el medio. 
móviles ${ }^{11}$, de acuerdo a los objetivos específicos de cada medio o programa. Tienden a abrir sus webs a las redes sociales y este proceso facilita la entrada a otros medios, aplicaciones y dispositivos digitales integrados en el grupo multimedia.

Asimismo, observamos que aunque cada uno de los grupos conserva sus peculiaridades, enfoques y planteamientos particulares, ambos mantienen un proceso similar en su penetración en las redes. Existen muchas semejanzas entre Atresmedia y $\mathrm{Me}$ diaset por ejemplo en sus actuaciones digitales, en cuanto a sus proyectos estratégicos de ofrecer contenidos en directo y a la carta, así como a la hora de querer establecer un vínculo interactivo con su audiencia.

Apreciamos que ambos grupos audiovisuales tienen muy en cuenta a su público y emplean las redes sociales como herramientas muy útiles para llegar a él. Con tal fin, enriquecen sus ofertas con otros datos y documentos adicionales para fomentar la interactividad. Buscan una mayor dinamización con sus seguidores lo que les obliga de manera casi constante a mejorar sus productos, innovar y conseguir fórmulas más creativas o sugerentes que atraigan su atención.

En este aspecto, cada grupo define su estrategia de atracción de su público de distinta forma, así mientras Mediaset no introduce tantos contenidos de pago en sus plataformas, aunque sí lo hace por el registro de usuarios a través de Facebook o por Medianet, Atresmedia apuesta por esta modalidad de pago para ofrecer ciertos servicios añadidos o de mayor calidad.

Igualmente percibimos que Atresmedia confía en sus aplicaciones "Atresmedia Conecta" y "Atresplayer ", a la par que Mediaset lo hace con las de sus programas más potentes, como "Gran Hermano" o "La Voz", o con la programación deportiva.

En definitiva, los dos grupos experimentan otras ofertas basadas en las sinergias con servicios auxiliares en sus plataformas digitales y están abiertos a nuevas y diferentes relaciones con la audiencia, en su intento de incrementar su valor interactivo y de mercado. Del mismo modo, tanto Atresmedia como Mediaset coinciden en afirmar que hasta ahora su mejor estrategia y la clave de haber asegurado su éxito han consistido en mantener un contenido distinto e interesante en sus plataformas. En nuestro análisis constatamos esta singularidad y especialidad en la oferta programática de cada canal en la competitividad por ampliar el número de seguidores, y con ello, como empresas privadas, con ánimo de lucro, lograr el incremento publicitario y de patrocinios, con el fin de alcanzar una rentabilidad económica por su esfuerzo.

En nuestra observación final con independencia a estas estimaciones y expectativas válidas y plausibles de ambos grupos, entendemos que todavía no existe un criterio definido y claro en la convergencia de diferentes actuaciones para la puesta en marcha de sus aplicaciones digitales ni en el empleo de las redes sociales como modo de acercamiento, de personalización de sus contenidos e interactividad con el usuarioconsumidor. Reconocemos que la innovación debe encontrarse en una diversidad de ofertas programáticas y en la apertura a una multiplicidad de posibilidades para el telespectador, y la opción de comentar y reenviar sus contenidos, informaciones o comentarios está muy poco explotada y lejos de alcanzar toda su potencialidad.

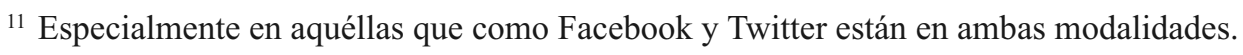


Asimismo, creemos que sería conveniente desarrollar nuevas estrategias de sinergias, de apoyos, de complementariedad más homogéneas, más sistemáticas entre los distintos medios y plataformas pertenecientes a estos grupos.

Por último, concluimos diciendo que la innovación tecnológica con Internet y la digitalización de la televisión deben suponer mayor calidad, originalidad y creatividad en las nuevas ofertas de contenidos. Lo que hasta ahora sólo significa innovación en el funcionamiento y adaptación a la tecnología emergente debe llegar a convertirse en un antes y un después, en un modelo comunicativo adecuado a este nuevo entorno de convergencia multimediática.

\section{Referencias bibliográficas}

BARCELÓ UGARTE, Teresa; GONZÁLEZ CONDE, M. Julia; y LÓPEZ VIDALES, Nereida (2014) "El uso del móvil por los jóvenes españoles, según el sexo”. Study on the use of mobile applications in Spanish youth in a gender. UK, Cambridge Scholars Publishing.

DOMINGO, David; SALAVERRÍA, Ramón; CABRERA, Miguel Ángel; y AGUADO, Juan Miguel (2007): "Convergencia de medios: dimensiones del debate sobre la disolución de los límites de los modelos periodísticos establecidos". IV Congreso Internacional de Comunicación y Realidad. Barcelona.

GONZÁLEZ CONDE, M. Julia (2009a): "Fórmulas y funciones de la expresión audiovisual en el campo semántico de lo cotidiano". Diccionarios y libros de estilo. Madrid, Fragua.

GONZÁLEZ CONDE, M. Julia (2009b): "La manipulación de la realidad en el discurso televisivo de la imagen" en Lengua y televisión. Madrid, Fragua.

GONZÁLEZ CONDE, M. Julia y BARCELÓ UGARTE, Teresa (2009): La televisión. Estrategia audiovisual. Madrid, Fragua.

ORIHUELA, José Luis (2008). "La hora de las redes sociales" en Nueva Revista, núm. 119, pp. 57-62.

SALGADO SANTAMARÍA, Carmen (2014): "Las redes sociales en las televisiones en España y en las cadenas de habla hispana de EE.UU", en GONZÁLEZ VALLÉS, Juan Enrique: POD - Comunicación actual: Redes sociales y lo 2.0 y 3.0. Madrid, McGraw-Hill.

SALGADO SANTAMARÍA, Carmen y GONZÁLEZ CONDE, M. Julia (2014): “Las redes sociales como plataformas de medios y servicios", en FLORES, Jesús: Contenidos y servicios periodísticos en las redes sociales. Madrid, Fragua.

SALGADO SANTAMARÍA, Carmen y ZAMARRA LÓPEZ, Mercedes (2014): “Sinergias periodísticas entre las redes sociales, los medios audiovisuales y la prensa digital", en CEBRIÁN HERREROS, Mariano: Innovaciones Periodísticas en las redes sociales. Madrid. UCM. 